\title{
Galactic Chemical Evolution
}

\author{
Brad K. Gibson, Yeshe Fenner, Agostino Renda, Daisuke Kawata \\ and Hyun-chul Lee
}

\begin{abstract}
Centre for Astrophysics \& Supercomputing, Swinburne University, Mail \#31, Hawthorn, Vic 3122, Australia bgibson,yfenner,arenda,dkawata,hclee@astro.swin.edu.au
\end{abstract}

Received 2003 September 2, accepted 2003 November 15

\begin{abstract}
The primary present-day observables upon which theories of galaxy evolution are based are a system's morphology, dynamics, colour, and chemistry. Individually, each provides an important constraint to any given model; in concert, the four represent a fundamental (intractable) boundary condition for chemodynamical simulations. We review the current state-of-the-art semi-analytical and chemodynamical models for the Milky Way, emphasising the strengths and weaknesses of both approaches.
\end{abstract}

Keywords: Galaxy: abundances — Galaxy: evolution — Galaxy: formation — Galaxy: kinematics and dynamics — methods: N-body simulations

\section{Introduction}

Stars and interstellar gas in galaxies exhibit diverse chemical element abundance patterns that are shaped by their environment and formation histories. The aim of Galactic Chemical Evolution (GCE) is to use the observed abundances to unlock earlier epochs in the Universe, probe the mechanisms of galaxy formation, and gain insight into the evolution of stellar systems.

Models for the chemical evolution of galaxies need to account for the collapse of gas and metals into stars, the synthesis of new elements within these stars, and the subsequent release of metal-enriched gas as stars lose mass and die. An additional feature of most models is the ongoing accretion of gas from outside the system. The most sophisticated models also incorporate a self-consistent treatment of the system's dynamics - both collisionless and dissipative components - either under idealised (semicosmological) conditions or within a full cosmological framework. Coupling GCE codes to a spectrophotometric evolution package further ensures that the models are constantly tested against real-world observational constraints.

Semi-analytic homogeneous models make simplifying assumptions that enable the mean trends of galactic systems to be calculated by numerically solving a set of equations governing the formation, destruction, and distribution of the elements as they cycle through gas and stars. One strength of these models is that they typically have the fewest number of free-parameters, making convergence to a unique solution more likely. A weakness of homogeneous models is the inherent assumption that stellar ejecta from dying stars is instantly mixed back into the ambient interstellar medium (ISM). Inhomogeneous GCE models relax this so-called 'instantaneous mixing approximation' in a semi-analytical manner, allowing consideration of observed trends in dispersion in various galactic observables. The self-consistent treatment of not only GCE, but the dynamics of a galaxy's gas, stars, and dark matter, remains the purview of chemodynamical codes. Each of the above are complementary tools for deconstructing the formation and evolution of systems such as our Milky Way: semi-analytical models can cover a range of parameter space that a chemodynamical code cannot, due to the many orders of magnitude difference in the respective computational demands, while the latter afford a coupling of the dynamics of the system to that of the GCE, in a manner not otherwise available.

In the review which follows, we present a biased overview of contemporary research in the field of GCE. For seminal reviews tracing the development and principles of this topic, the reader is referred to Tinsley (1980), Matteucci (2001), and references therein. Section 2 summarises the most popular formation and evolutionary scenarios and describes the relationships between different components of the Galaxy. Principles of homogeneous and inhomogeneous semi-analytical models are presented in Section 3 and Section 4, respectively, while in Section 5, the state of the art in three-dimensional cosmological chemodynamical codes is reviewed. Coupling the GCE predictions from both of these approaches to galaxy evolution to the colour and luminosity information provided by spectrophotometric codes is discussed in Section 6 . Potential future areas of interest to the field are itemised in Section 7.

\section{Structure and Formation of the Galaxy}

The thin disk and bright inner bulge are the brightest components of the Milky Way. They are housed within a much more extended and diffuse spheroidal stellar halo - as distinct from the dark matter halo. In addition, a fourth main component - the thick disk — was identified by Gilmore \& Reid (1983). The latter found that star counts obtained by an extensive UK Schmidt photometric survey were well-fitted by a multi-component stellar population model consisting of (1) a thin disk, (2) thick disk, and (3) halo. This three-component model breaks down within the central kiloparsec of the Galaxy where a dense metal-rich 
Table 1. Milky Way properties

\begin{tabular}{lcccc}
\hline & $\begin{array}{c}\text { Mean } \\
\text { age }(\mathrm{Gyr})^{a}\end{array}$ & $\begin{array}{c}\text { Mean } \\
{[\mathrm{Fe} / \mathrm{H}]^{a}}\end{array}$ & $\begin{array}{c}\text { Scale height } \\
(\mathrm{kpc})\end{array}$ & $\begin{array}{c}\text { Scale length } \\
(\mathrm{kpc})\end{array}$ \\
\hline Halo & 14 & -1.78 & Effective radius $\sim 2.7^{b}$ \\
Thick disk & 11 & -0.78 & $\sim 0.75^{c}$ & $3.5^{c}$ \\
Thin disk & $5-7$ & -0.14 & $\sim 0.33^{c}$ & $2.25^{c}$ \\
Bulge & 10 & 0 & Effective radius $\sim 1.2^{d}$ \\
\hline
\end{tabular}

${ }^{a}$ Robin et al. (2003)

${ }^{b}$ de Vaucouleurs profile - Buser et al. (1998)

${ }^{c}$ Chen et al. (2001)

${ }^{d}$ de Vaucouleurs profile — Yoshii \& Rodgers (1989)

bulge begins to dominate (Gilmore, Wyse, \& Kuijken 1989). The thick disk component has an exponential scale height of $1350 \mathrm{pc}$ - about four times greater than the scale height of the thin disk - and comprises 2\% of the nearby stars. Photometric studies of external galaxies had already established that thick disks are common to spirals (van der Kruit \& Searle 1982). A popular explanation for the presence of thick disks is that mergers with smaller satellites during early times heated the thin disk (Wyse \& Gilmore 1993).

In order to distinguish individual populations of stars, one wishes to know not just their spatial distribution, but their kinematics, chemical abundances, and ages. A complete dataset of this information should enable one to reconstruct the formation and evolution of the Milky Way. A wealth of past, present, and future surveys and satellite missions (e.g. HIPPARCOS, ${ }^{1}$ RAVE, ${ }^{2}$ GAIA $^{3}$ ) offer (or will offer) the opportunity to determine the order in which the Galactic components formed, whether they evolved independently of one another, and how important merging has been in assembling the Milky Way. The mean age and metallicity of the halo, thick disk, thin disk, and bulge are shown in Table 1.

Traditionally two scenarios have competed to explain the formation of the Milky Way:

1. The first scenario, proposed by Eggen, Lynden-Bell, \& Sandage (1962), describes the rapid monolithic collapse of a protogalactic gas cloud to form the halo. The Galactic disk would have subsequently formed as the residual gas dissipationally collapsed. This would naturally give rise to two populations of stars: an older, more metal-poor group found in the halo, and a younger, more metal-rich group orbiting closer to the Galactic mid-plane.

2. Searle \& Zinn (1978) offered an alternative to the monolithic collapse picture, proposing that the Galaxy was constructed from smaller cloud fragments, in which stars may have already started forming.

The Galaxy's true formation history is likely to lie somewhere between the two extremes of primordial collapse

\footnotetext{
${ }^{1} \mathrm{http}: / /$ astro.estec.esa.nl/Hipparcos/

${ }^{2}$ http://astronomy.swin.edu.au/RAVE/

${ }^{3} \mathrm{http}: / /$ astro.estec.esa.nl/GAIA/
}

and hierarchical formation. Chemical properties of stars provide important clues into disentangling the puzzle of the Galaxy's formation. The relative abundances of certain elemental species act as 'cosmic clocks', by which the formation timescales of various stellar populations can be determined. A popular cosmic clock is the ratio of an element like oxygen, which is born mostly in massive, very short-lived stars, and an element like iron, whose creation is linked to lower mass longer-lived progenitors (Gilmore et al. 1989).

\section{Homogeneous Models}

Homogeneous GCE models have traditionally formed the cornerstone of this field and consquently have a rich literature to draw upon. The basic ingredients, observational constraints, and several weaknesses are highlighted below, although it should be emphasised that much of this discussion pertains also to the inhomogeneous and chemodynamical models described in Sections 4 and 5 (as many of the ingredients are common to all techniques).

\subsection{Basic Ingredients}

The main ingredients of homogeneous GCE models are outlined below and we discuss their relationship to one another through the basic set of chemical evolution equations.

- Stellar yields and lifetimes: Almost all elements heavier than helium originate from stars. Stars enrich the ISM with their own unique pattern of elements depending on their mass and initial metallicity. The predicted stellar yields consequently form the backbone of the study of GCE. The grids of yields utilised in the models are the outcome of computations of stellar evolution and vast networks of nuclear reactions. For the purposes of chemical evolution, stars are often divided into three categories:

- Massive stars $\left(m \gtrsim 10 \mathrm{M}_{\odot}\right)$ evolve quickly because their enormous gravitational potential accelerates the nucleosynthesis process. Their death is marked by a violent supernova ( $\mathrm{SN})$, leaving behind a neutron star or black hole. Although massive stars are much rarer than their lower mass counterparts, they are the main source of most of the heavy elements (i.e. metals) in the Galaxy. Figure 1 shows the production factors from massive stars predicted by the detailed nucleosynthesis calculations of Woosley \& Weaver (1995, left panel) and the FRANEC code (kindly provided by A. Chieffi 2003, personal communication; FRANEC is described in Chieffi \& Limongi 2002, right panel). ${ }^{4}$ The dotted line at $[\mathrm{X} / \mathrm{O}]=0$ indicates the solar elemental abundance pattern relative to $\mathrm{O}$. The solar abundance pattern of most metals is adequately reproduced by massive stars but $\mathrm{C}, \mathrm{N}$ and the iron-peak elements require additional production sites.

\footnotetext{
${ }^{4}$ A generic comparison of massive star yields can be found in Gibson, Loewenstein, \& Mushotzky (1997).
} 

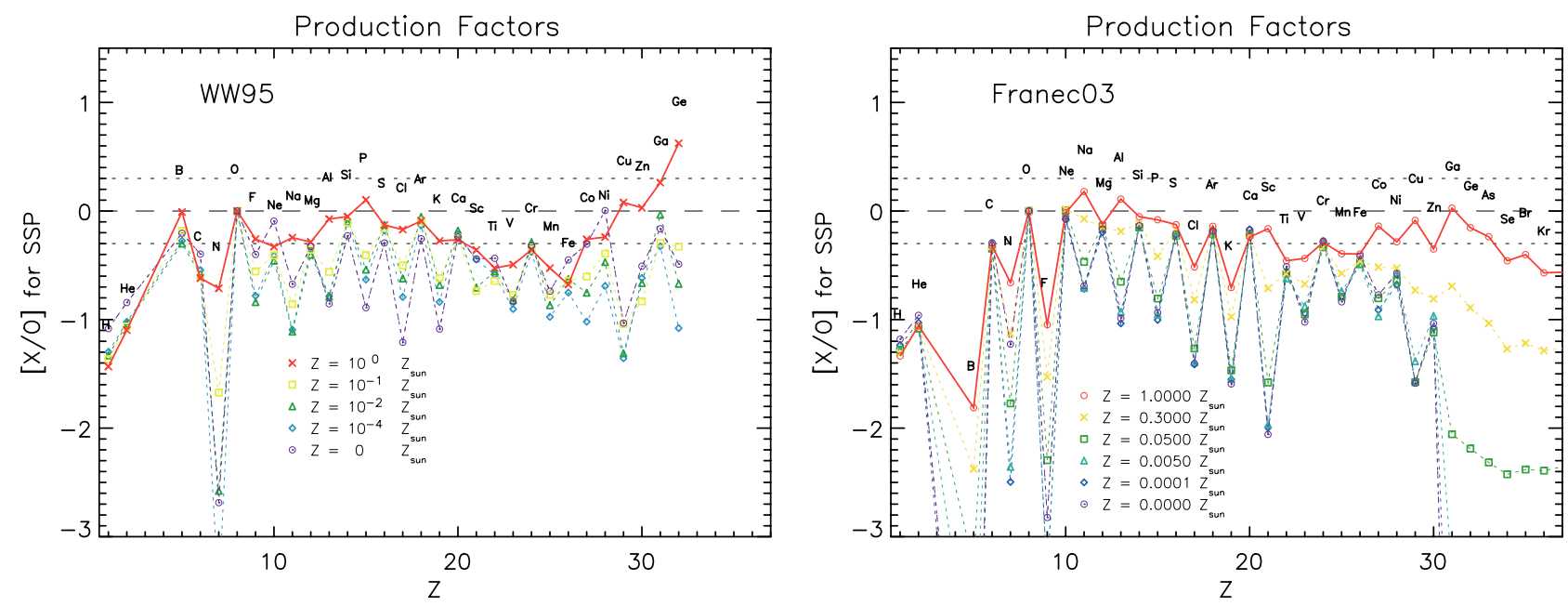

Figure 1 Production factors relative to $\mathrm{O}$ on a solar logarithmic scale from a single generation of massive stars using the metallicity-dependent yields of Woosley \& Weaver 1995 (left panel) and those of FRANEC 2003 (right panel). The latter were kindly provided by A. Chieffi (2003, personal communication). Yields were integrated over a Salpeter (1955) IMF from 12 to $40 \mathrm{M}_{\odot}$. The dashed line indicates the solar values (where $\log \left(\mathrm{N}_{\mathrm{O}} / \mathrm{N}_{\mathrm{H}}\right)_{\odot}+12=8.73$, Holweger 2001) and dotted lines indicate deviations from scaled solar by a factor of two. For both sets of yields $\mathrm{C}, \mathrm{N}$, and some of the iron-peak elements are subsolar because they require additional sources such as lower mass stars and Type Ia SNe. The strength of the 'odd-even' effect increases with decreasing metallicity in both cases, however the effect is more pronounced for FRANEC 2003.

- Intermediate- and low-mass stars (ILMS) live longer than their massive star counterparts, due to their lower density. They greatly outnumber the heavier stars but do not produce significant quantities of many elements besides helium, carbon, nitrogen, and certain isotopes, which are created through hydrostatic burning and expelled in stellar winds and planetary nebulae (e.g. van den Hoek \& Groenewegen 1997). Very low mass stars $\left(m \lesssim 1 \mathrm{M}_{\odot}\right)$ have lifetimes comparable to the age of the Galaxy and therefore serve to lock up the gas supply.

- ILMS in binary systems may culminate in powerful supernovae explosions classed as Type Ia. The exact physical mechanisms behind SNe Ia are still an open question but one popular theory holds that the mass lost by a binary star as it evolves is accreted by its smaller white dwarf (WD) companion until the WD can no longer be sustained by electron degenerate pressure. Then the entire mass of the WD is ejected in a violent explosion that converts much of the stellar material into iron (e.g. Iwamoto et al. 1999).

- Initial mass function (IMF): The precise form of the IMF dictates the number of stars born in a given mass interval in each generation of stars. This in turn sets the rate at which different elements are released into the ISM, thus influencing both the relative and absolute elemental abundances. Most IMFs in the literature consist of a simple single- or multi-component power law specified over a mass range from $m \sim 0.1 \mathrm{M}_{\odot}$ to an upper mass limit typically between 40 and $100 \mathrm{M}_{\odot}$ (e.g. Salpeter 1955; Scalo 1986; Kroupa, Tout, \& Gilmore 1993).

- Star formation rate (SFR): While laws of star formation can be calculated from first principles, chemical evolution models invariably use a functional form that has been derived empirically. An ample supply of gas is the first condition needed for star formation, so it is not surprising that one of the simplest laws has $\mathrm{SFR} \propto \sigma_{\text {gas }}^{k}$, where $\sigma_{\text {gas }}$ is the surface density of gas and the exponent $k$ may range from 1 to 2 (Schmidt 1959). Other star formation laws presume that factors such as total mass density and/or Galactocentric radius play a role. Dopita \& Ryder (1994) found that a law given by $\mathrm{SFR} \propto \sigma_{\text {gas }}^{5 / 3} \sigma_{\text {total }}^{1 / 3}$ satisfactorily describes the correlation between $\mathrm{H} \alpha$ emission and I-band surface brightness in spiral galaxies.

- Gas flows: In the simplest scenario, our model Galaxy in each radial bin can be considered a closed box consisting of primordial gas from which stars are born according to the chosen SFR and IMF prescriptions. In the classic closed-box model (e.g. Pagel \& Patchett 1975 ) there is no gas loss or gain; at time $=0$ all Galactic matter is present as primordial gas from which stars immediately form. This type of model, characterised by an intense period of early star formation, provides a reasonable account of the formation of the halo and bulge of the Milky Way. When applied to the Galactic disk, however, the basic closed-box model leads to an excess of metal-poor stars with respect to the observed metallicity distribution of nearby long-lived stars: the so-called 'G-dwarf problem' (Pagel \& Patchett 1975). More realistic models overcome this problem by allowing the Galactic disk to form via continual accretion of gas. The infall rate as a function of radius $r$ and time $t$ often takes the form

$$
\frac{\mathrm{d}}{\mathrm{d} t} \sigma_{g a s}(r, t)=A(r) e^{-t / \tau(r)},
$$

where $\tau(r)$ is the exponential infall timescale and the coefficient $A(r)$ must satisfy the constraint that 
$\int_{0}^{t_{\text {now }}} A(r) e^{-t / \tau(r)}=\sigma_{\text {gas }}(r)$, where $\sigma_{\text {gas }}(r)$ is the presentday surface density profile. Many elliptical and dwarf galaxies are expected to have undergone periods in which the energy from supernova explosions exceeds the gravitational energy of the system, causing the interstellar gas to be expelled in a galactic wind (Larson 1974; Dekel \& Silk 1986; Matteucci \& Tornambé 1987; Gibson 1997; Recchi, Matteucci, \& D'Ercole 2001). However, the outflow of gas is not expected to feature in the history of most spiral galaxies and is usually neglected in the models. Radial flows of gas have also been investigated within the homogeneous GCE paradigm (e.g. Portinari \& Chiosi 2000).

- Galactic components: The infall of gas described above might occur in multiple episodes that correspond to the formation of the individual halo, bulge, thick disk and thin disk components. Recent works have adopted different premises regarding the extent to which these components interact with each other through gas exchange. For instance, enriched halo gas might be funnelled into the bulge or else might collapse further to form the disk. Alternatively, the halo and thin disk might evolve coevally yet independently. The answer to these questions is partly clouded by challenges in distinguishing the various stellar populations. The properties that we wish to measure in each population, such as metallicity and kinematics, are also the properties used to define the populations.

\subsubsection{The Equations}

The chemical composition of the interstellar medium as a function of time and Galactocentric radius is described by equations that balance: (1) processes that deplete chemical elements from the interstellar medium, namely star formation and perhaps Galactic winds; and (2) processes that replenish the ISM, such as stellar winds, SNe, and infalling gas. The GCE equations can be solved analytically given the assumption that stars release their ejecta instantaneously at the time of their birth. This approximation is reasonable only for very massive and short-lived stars and it precludes one from reproducing the relative trends of elements that are restored to the ISM by different mass stars on different characteristic timescales. For GCE models to have the power to interpret the variation of diagnostic abundance ratios, the 'instantaneous recycling approximation' must be relaxed. There is another assumption, however, that few GCE models relax: namely, that the stellar yields are instantaneously mixed into the ISM. For this reason, homogeneous GCE models are not the ideal tools for studying the Galaxy in its infancy, when chemical abundances in the ISM could be traced to individual supernovae (or for present-day observables that originate from that era, e.g. the Galactic halo). We return to this assumption in Section 4.

Standard models of spiral galaxy chemical evolution assume azimuthal symmetry and collapse the object into a flat disk such that radius is the only spatial variable. Defining $\sigma_{i}(r, t)$ as the mass surface density of species $i$ at radius $r$ and time $t$, then the rate of change of $\sigma_{i}(r, t)$ is given by

$$
\begin{aligned}
\frac{\mathrm{d}}{\mathrm{d} t} \sigma_{i}(r, t)= & \int_{m_{\text {low }}}^{m_{\text {up }}} \psi\left(r, t-\tau_{m}\right) Y_{i}\left(m, Z_{t-\tau_{m}}\right) \frac{\phi(m)}{m} \mathrm{~d} m \\
& +\frac{\mathrm{d}}{\mathrm{d} t} \sigma_{i}(r, t)_{\text {infall }} \\
& -X_{i}(r, t) \psi(r, t) \\
& -\frac{\mathrm{d}}{\mathrm{d} t} \sigma_{i}(r, t)_{\text {outflow }}
\end{aligned}
$$

where the four terms on the right-hand side of equation (2) correspond to the stellar ejecta, gas infall, star formation, and gas outflow rates, respectively. $\psi$ is the SFR, $Y_{i}(m$, $Z_{t-\tau_{m}}$ ) is the stellar yield of $i$ (in mass units) from a star of mass $m$ and metallicity $Z_{t-\tau_{m}}, \phi(m)$ is the IMF by mass, and $X_{i}$ is the mass fraction of element $i$. By definition, the sum of $X_{i}$ over all $i$ is unity, and the total surface mass density is identical to the integral over the infall and outflow rates. $m_{\text {low }}$ and $m_{u p}$ are the lower and upper stellar mass limits, respectively, and $\tau_{m}$ is the lifetime of a star of mass $m$. In practice, the first term is split into three equations that deal separately with ILMS, Type Ia SNe progenitors, and massive stars (see Greggio \& Renzini 1983 and Matteucci \& Greggio 1986 for details).

\subsection{Observational Constraints}

The most thoroughly observed and best understood galaxy is the Milky Way, and in particular, the 'local' solar neighbourhood. So extensive is the Milky Way dataset, many model ingredients can be well-constrained empirically. Thus our own Galaxy is often the gauge by which chemical evolution models are calibrated. Indeed, studies of the cosmic evolution of disk galaxies often adopt scaling laws based on the Milky Way (e.g. Boissier \& Prantzos 2000). The extent to which the Milky Way's IMF, star formation law, and nucleosynthetic behaviour can be applied to other types of objects at earlier epochs depends on how universal these prescriptions are and on whether we live in a prototypical galaxy. A minimal set of observational constraints for GCE models is described below.

- Solar abundance pattern: Any chemical evolution model should be able to reproduce the solar abundance pattern, i.e. the pattern in the ISM $4.5 \mathrm{Gyr}$ ago at the radius where the Sun was born. The Sun is the single star with the most complete set of abundance measurements. For consistency, one can also compare the chemical composition of solar system meteorites with estimates based on stellar spectral lines. As discussed above, the predicted solar enrichment pattern is chiefly controlled by the yields released in (1) Type II SN explosions of massive stars, (2) planetary nebulae and stellar winds of ILMS, and (3) Type Ia SN explosions of binary systems of ILMS. In addition, the predictions are sensitive to the stellar mass distribution (i.e. the IMF) and the SFR, since these set the relative contribution and enrichment timescale from different types of stars. A 
seminal attempt to simulate the evolution of all elements up to and including zinc was made by Timmes, Woosley, \& Weaver (1995) using the comprehensive grid of massand metallicity-dependent yields calculated by Woosley \& Weaver (1995). Impressively, Timmes et al. (1995) reproduced the abundances of most isotopes in the Sun to within a factor of two.

It is worth noting that even our understanding of the solar abundance pattern still has the power to surprise us. As recently as a decade ago, the solar oxygen abundance was assumed to be $\log \left(\mathrm{N}_{\mathrm{O}} / \mathrm{N}_{\mathrm{H}}\right)_{\odot}+12=8.93$ (Anders \& Grevesse 1989); it has become clear recently though that accounting for solar granulation and nonLTE effects properly leads to a significantly radical downward revision in the Sun's oxygen abundance by almost a factor of two, to $\log \left(\mathrm{N}_{\mathrm{O}} / \mathrm{N}_{\mathrm{H}}\right)_{\odot}+12=8.69$ (Allende Prieto, Lambert, \& Asplund 2001). Such a shift partially resolves the long-standing dichotomy between the Sun's oxygen abundance and that of the local ISM.

- G-dwarf distribution: A much stronger constraint on GCE models is the distribution of stars as a function of metallicity, since this represents the convolution of the age-metallicity relationship and the star formation history. In order to probe the early Galaxy, one needs a sample of low mass stars such as G- or K-dwarfs whose main-sequence lifetimes are comparable to, or greater than, the age of the Universe. GCE models have demonstrated that the paucity of low-metallicity dwarf stars can be explained if the Galactic halo formed first on a rapid timescale, followed by a slow build-up of the thin disk (e.g. Chiappini, Matteucci, \& Gratton 1997; Alibés, Labay, \& Canal 2001; Fenner \& Gibson 2003). The excess of metal-poor stars predicted by simple closed-box models can also be avoided by assuming prompt initial enrichment, perhaps by a first generation of extremely massive Population III stars. If this were the case, then the abundance pattern of Population III ejecta should be evident in the lowest metallicity stars. It has also been suggested that there are no very metal-poor stars left because they all had relatively short lifetimes due to low metallicity environments favouring the formation of higher mass stars (e.g. Nakamura \& Umemura 2001). A further consideration is that an initially pristine zero-metallicity star might have had its surface layers polluted by the accretion of metals from the ISM over the past $\sim 12$ Gyr (e.g. Shigeyama, Tsujimoto, \& Yoshii 2003).

- Evolution of abundance ratios: If elements $X_{1}$ and $\mathrm{X}_{2}$ have different origins and different characteristic timescales for release into the ISM, then $\left[\mathrm{X}_{1} / \mathrm{X}_{2}\right]$ vs $\left[\mathrm{X}_{2} / \mathrm{H}\right]$ acts as a clock by which chemical evolution can be measured (e.g. Wyse \& Gilmore 1988). For example, readily observable features of oxygen and iron in stellar spectra have encouraged the wide use of $[\mathrm{O} / \mathrm{Fe}]$ vs $[\mathrm{Fe} / \mathrm{H}]$ to diagnose the overall star formation history of galactic systems. As with most heavy elements, oxygen is produced chiefly in massive and short-lived stars. Thus, oxygen enrichment immediately follows the onset of star formation. In contrast, at least half of the iron in the Galaxy probably originated from Type Ia SNe (e.g. Alibés et al. 2001), whose lower-mass and longer-lived progenitors introduce a time delay for iron enrichment. The remaining iron comes largely from Type II SNe. The combination of high $[\mathrm{Fe} / \mathrm{H}]$ and high $[\mathrm{O} / \mathrm{Fe}]$ is understood to arise in systems that formed stars so rapidly that high metallicities were reached before $\mathrm{SNe}$ Ia had a chance to lower the $[\mathrm{O} / \mathrm{Fe}]$ value (Smecker-Hane \& Wyse 1992). Similarly, one might interpret low $[\mathrm{Fe} / \mathrm{H}]$ and low $[\mathrm{O} / \mathrm{Fe}]$ as a sign of a slowly evolving system.

- SFR and SN rates: The present-day star formation and Type II and Type Ia SNe rates must be matched by a successful chemical evolution model. However these are fairly weak constraints given that we can only be reasonably certain about the current SFR and mean past rate. Finer details of the Galactic star formation history (SFH) are difficult to recover and are quite uncertain. The most direct way to infer the SFH is by determining the age distribution of stars; a method that relies upon unreliable stellar ages and assumptions about the IMF, stellar evolution, scale height corrections, and stellar kinematics (Rocha-Pinto et al. 2000a). This technique is also somewhat circular, in that a SFH must have been assumed in order to derive the IMF.

- Age-metallicity relationship: This is an important constraint, but again, a weak one given that the scatter in the observations (e.g. Ibukiyama \& Arimoto 2002) can accommodate most model predictions. Moreover, the very existence of an AMR, which had been well established by earlier studies (e.g Twarog 1980; Edvardsson et al. 1993; Rocha-Pinto et al. 2000b), has recently been challenged by investigations demonstrating large intrinsic scatter and no significant trend of metallicity with age (e.g. Feltzing, Holmberg, \& Hurley 2001).

- Gas and abundance gradients: It has long been known that the Milky Way is more metal-rich toward its centre and more metal-poor at large Galactocentric distance (Tinsley 1980, and references therein). Using the oxygen abundance observed in H II regions and OB stars to trace metallicity, a metallicity gradient of $-0.07 \mathrm{dex} \mathrm{kpc}^{-1}$ has been established (e.g. Smartt \& Rolleston 1997). The abundance of metals in a region of gas is particularly sensitive to the balance between the star formation and gas accretion rates. Therefore the predicted metallicity gradient of the Galactic disk depends strongly on how the star formation prescription and gas infall rate are assumed to vary with radius. Good fits to the data are obtained by 'inside-out' formation scenarios, whereby the innermost disk is built-up on the shortest timescale (e.g. Larson 1976; Chiappini et al. 1997). Portinari \& Chiosi (1999) showed that a SFR such as the Schmidt (1959) law, which varies only with the gas surface density, produces a radial abundance profile that is too flat unless one assumes an unreasonably large variation in formation timescale 
from the inner to outer disk such that the far disk would currently be accreting at much higher rates than observed. The Dopita \& Ryder (1994) law, with a mild dependence on total mass surface density, yields a better fit to the metallicity gradient (Portinari \& Chiosi 1999). The theoretical metallicity distribution of long-lived stars as a function of Galactocentric radius is shown in Figure 2, assuming a Dopita \& Ryder (1994) star formation law.

- Isotopic abundances: Traditionally, chemical evolution studies have been concerned with monitoring the total abundance (or dominant isotope) of specific elements in order to unravel the Galaxy's history. Recent advances in instrumentation have paved the way for research into individual isotopes that provides new challenges for nucleosynthesis theory. For instance, Type II SNe models appeared capable of explaining the magnesium isotopic ratios in intermediate to solar metallicity stars, but the results from the solar neighbourhood model shown in Figure 3 reveal an underproduction of ${ }^{26} \mathrm{Mg} /{ }^{24} \mathrm{Mg}$ at low metallicites (dotted line) with respect to the latest data. The missing piece of the puzzle may be low metallicity intermediate mass stars on the asymptotic giant branch (AGB), whose helium hells may be hot enough to generate ${ }^{25} \mathrm{Mg}$ and ${ }^{26} \mathrm{Mg}$ by triggering $\alpha$-capture onto ${ }^{22} \mathrm{Ne}$ (Karakas \& Lattanzio 2003). As Fenner et al. (2003) have demonstrated for the first time, the data at $[\mathrm{Fe} / \mathrm{H}] \lesssim-1$ are much better matched after incorporating the Karakas \& Lattanzio (2003) AGB nucleosynthesis calculations in a chemical evolution model (solid line). Such detections of isotopic ratios in field stars and globular clusters may reveal a great deal about the relative role of different types of stars in various environments.

\subsection{Uncertainties and Weaknesses}

- Iron-peak yields: Iron-peak elements are buried deep within the cores of massive stars near the radius that separates the ejected material from the remnant. The location of this so-called 'mass cut' is a free parameter in stellar models, one which controls the relative abundances of the iron-peak elements as well as the $\mathrm{X}_{i} / \mathrm{Fe}$ ratio in the ejecta. Abundances in very metal-poor stars can help constrain the choice of mass cut, however in order to simultaneously eject iron-peak elements in the correct proportions and recover the high observed $[\alpha / \mathrm{Fe}]$ ratios, models need to incorporate mixing and fallback (Umeda \& Nomoto 2002) or asymmetrical explosions. Multi-dimensional simulations of explosive nucleosynthesis may reveal more about these processes (Travaglio, Kifonidis, \& Müller 2003).

- Shape and evolution of the IMF: A time-invariant IMF remains the best choice for modelling the general evolution of our own Galaxy (Chiappini, Matteucci, \& Padoan 2000), but peculiar abundance patterns in extremely metal-poor stars support the notion that the first generation of stars was biased towards higher masses (Chieffi \& Limongi 2002). The upper limit of the

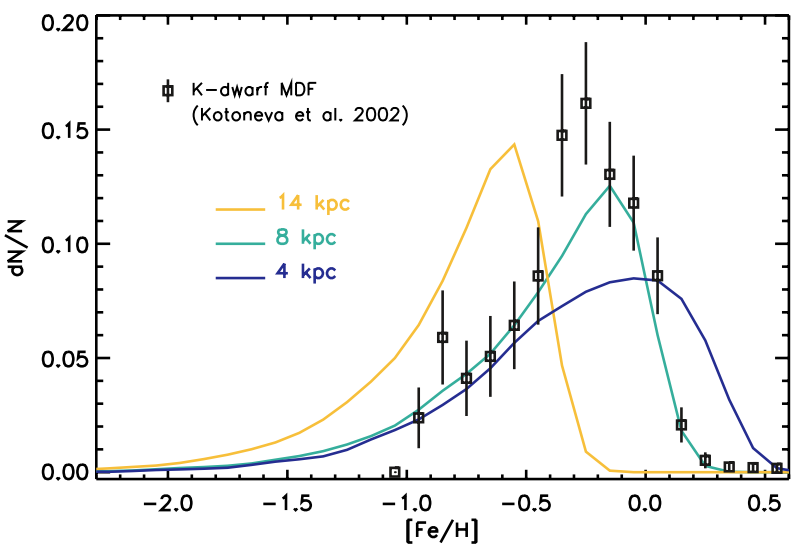

Figure 2 The metallicity distribution function (MDF) of long-lived stars predicted by a single infall model at three different Galactocentric radii: 4,8 , and $14 \mathrm{kpc}$. Open squares with error bars correspond to the MDF of nearby K-dwarfs (i.e. at a radius $\sim 8-8.5 \mathrm{kpc}$ ) observed by Kotoneva et al. (2002). The theoretical MDF has been convolved with a Gaussian of dispersion $\sigma=0.1 \mathrm{dex}$ in $[\mathrm{Fe} / \mathrm{H}]$ to simulate the empirical uncertainties.

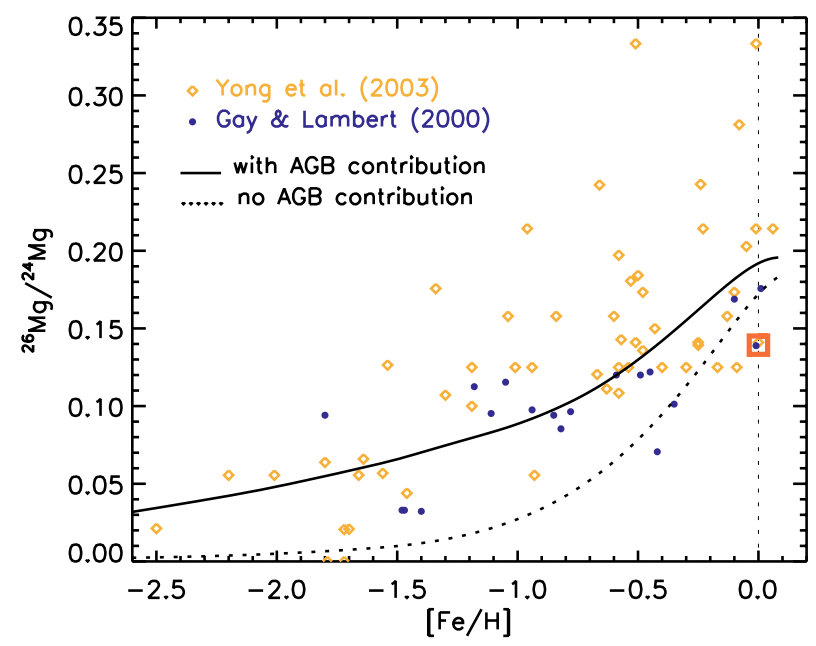

Figure 3 The predicted metallicity evolution of magnesium isotopic ratio ${ }^{26} \mathrm{Mg} /{ }^{24} \mathrm{Mg}$ for the solar neighbourhood both with (solid curve) and without (dotted curve) a contribution from intermediatemass AGB stars (from Fenner et al. 2003). Stellar data are from Gay \& Lambert (2000, blue circles), and data for halo and thick disk stars are from D. Yong et al. (in preparation, orange diamonds). The red square denotes the solar ratio.

stellar IMF, $m_{u p}$, is also uncertain and impacts upon the total amount of metals produced by each stellar generation. Figure 4 illustrates the sensitivity of metal growth in the solar neighbourhood to the upper IMF mass. Increasing $m_{u p}$ from $40 \mathrm{M}_{\odot}$ to $100 \mathrm{M}_{\odot}$ is expected to raise the metallicity by as much as $30 \%$. This is due to the steeply increasing yield of $\mathrm{O}$ (which is the most abundant metal in the interstellar gas) as a function of stellar mass.

- Black hole mass limit: Essentially a free parameter in Type II SNe models, the mass above which most of the stellar material collapses to form a black hole must be addressed a posteriori, in an empirical 


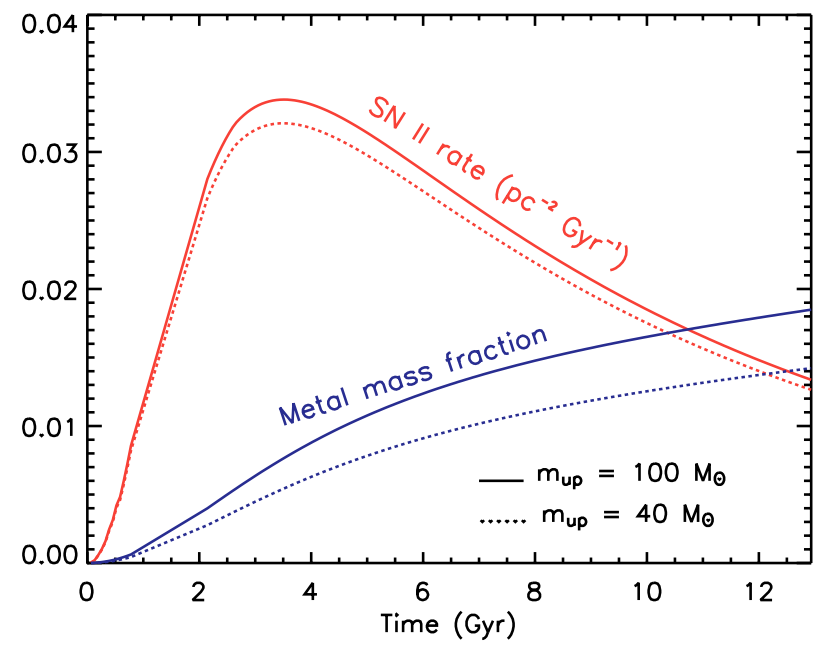

Figure 4 Evolution of Type II SN rate (red lines) and metallicity (blue lines) predicted by a single infall phase model of the solar neighbourhood for an IMF upper mass limit of $40 \mathrm{M}_{\odot}$ (dotted lines) and $100 \mathrm{M}_{\odot}$ (solid lines). Metallicity is defined as the mass fraction of elements heavier than He in the interstellar medium (ISM). The SN II rate is barely affected by changes in the upper limit to the (Kroupa et al. 1993) IMF, however metallicity is about $30 \%$ higher for the $m_{u p}=100 \mathrm{M}_{\odot}$ case. This reflects the steep increase in the yield of $\mathrm{O}$ (which is the dominant element contributing to metallicity) as a function of initial stellar mass.

manner (to recover the observable constraints alluded to earlier). Black hole progenitors are expected to release most of their oxygen, carbon, etc., via pre-SN stellar winds, but heavier elements such as iron are expected to fall back onto the remnant. One may expect the mass range corresponding to black hole collapse is sensitive to metallicity (e.g. Maeder 1992).

- Limited dataset: Many conclusions about cosmic chemical evolution have been drawn in part from studies of the Milky Way, but the Milky Way is only one object. How unique or typical the Milky Way is amongst other galaxies is unknown and it can be dangerous to take the Milky Way's evolutionary path as representative of most spirals. For instance, a system such as M31, which resembles the Milky Way in terms of size and morphology, shows evidence for dramatically different metallicity distributions of its stellar populations (Worthey \& Espana 2003). As an example of the perils of drawing conclusions based on the solar neighbourhood, the almost constant ratio of $\mathrm{Zn} / \mathrm{Fe}$ versus metallicity might be taken as a sign that zinc, like iron, owes much of its production to Type Ia SNe (Matteucci et al. 1993; Mishenina et al. 2002). It is possible, however, that zinc comes primarily from Type II SNe, with a strongly metallicity-dependent yield that mimics the time delay associated with a SN Ia source (e.g. Timmes et al. 1995). As a consequence, zinc would not always vary in lockstep with iron but would depend on specific formation histories. It is worthwhile to bear this in mind, as zinc is often used as a proxy for iron in the high-redshift universe.

\section{Inhomogeneous Models}

The halo has special significance for the formation of the Galaxy. If halo stars form a collisionless system, their orbits contain information about the dynamics at the time of the formation. In addition, the abundances observed in low mass stars reflect the chemical abundances and inhomogeneities during halo formation. Element abundance ratios as a function of metallicity (generally) show increasing scatter with decreasing metallicity, ${ }^{5}$ whereas at higher metallicities the scatter decreases to reach a mean element abundance which corresponds to the ratio of the stellar yields integrated over the IMF (the regime in which the homogeneous GCE models of Section 3 are most applicable).

The enrichment of the halo mainly depends upon the number of SN explosions and the manner in which the ejected gas is mixed with the ambient ISM. If the mixing volume is sufficiently large, the enrichment could be spatially homogeneous. Conversely, should mixing be inefficient, significant (localised) abundance inhomogeneities could exist; in theory, gas in the vicinity of a SN might even bear the chemical imprint of that single event. If the latter were the case, second-generation, extremely metal-poor halo stars may show an abundance pattern which matches the nucleosynthetic yields of a single Population III SN. A highly inhomogeneous halo would naturally result in this case. Simulating the temporal and spatial history of chemical inhomogeneities in the halo of the Milky Way, through semi-analytical GCE models, is an extremely active field at the present. Important earlier work in this field includes investigations by Malinie, Hartmann, \& Mathews (1991), Malinie et al. (1993), Pilyugin \& Edmunds (1996), Copi (1997), van den Hoek \& de Jong (1997), Ikuta \& Arimoto (1999), and Travaglio, Galli, \& Burkert (2001). In what follows, we review three of the more recent approaches to modelling inhomogeneous chemical evolution.

\subsection{Argast et al. $(2000,2002)$}

Argast et al. (2000, 2002) evolve an $\sim 15 \mathrm{kpc}^{3}$ region of mass $10^{8} \mathrm{M}_{\odot}$ with a spatial resolution of $50 \mathrm{pc}$. At each time step (1 Myr), a certain number of cells are randomly chosen, and each one can create a star with a probability proportional to $\sigma_{\text {gas }}^{2}$ (akin to the Schmidt Law described in Section 3.1), under the assumption of a Salpeter (1955) IMF. Type Ia SNe were not included in their models, and the simulations were halted once $[\mathrm{Fe} / \mathrm{H}]$ reached $1 / 10$ solar. Stars which form out of material enriched by a single $\mathrm{SN}$ will inherit its abundances and show an elemental pattern which reflects the progenitor mass. The Argast et al. (2000) model has minimal ISM mixing, as the expansion of the SN remnant is the only dynamical process taken into account.

The results of their Inhomogeneous Galactic Chemical Evolution (IGCE) modelling — in relation to

\footnotetext{
${ }^{5}$ As Spite et al. (2003) note though, this canonical 'wisdom' needs some revision.
} 
the trend of abundance ratio scatter as a function of $[\mathrm{Fe} / \mathrm{H}]$ - are in good agreement with observations, except for $[\mathrm{Cr} / \mathrm{Fe}],[\mathrm{Mn} / \mathrm{Fe}]$, and $[\mathrm{Ni} / \mathrm{Fe}] .{ }^{6}$ Argast et al. conclude that for $[\mathrm{Fe} / \mathrm{H}]<-3$, the ISM is unmixed and dominated by local inhomogeneities polluted by individual SN events. For $[\mathrm{Fe} / \mathrm{H}]>-2$, their model halo ISM reflects a true IMF-averaged abundance pattern and is considered 'well-mixed'. Argast et al. do note though that an individual SN event can still have an impact upon a well-mixed ISM, leading to finite dispersions in abundance patterns at disk-like metallicities.

\subsection{Oey $(2000,2003)$}

The initial condition of Oey's (2000) IGCE model is that of a metal-free closed box, in which the first generation of star forming regions is randomly distributed, occupying a volume filling factor $Q$. Each region behaves as a superbubble powered by its enclosed Type II SNe. Oey then considers $n$ subsequent generations of star formation, allowing the star forming regions to overlap. The main conclusion drawn by Oey (2000) is that the evolutionary state of a system is characterised by the product $n Q$, with the relative filling factor of contamination having the same importance as the number of contaminating generations. The high metallicity tail of the MDF may provide a useful discriminant between the classical 'Simple Model' and the Simple Inhomogeneous Model proposed by Oey. The latter agrees with both the Galactic halo and bulge MDFs by varying only this single parameter $n Q$, with $Q$ and $n$ independent and roughly associated with the global star formation efficiency and age.

The Simple Inhomogeneous Model assumes no largescale mixing beyond the superbubble radii, with metals uniformly distributed within the volumes of the hot superbubbles and cooling locally. Once mixing is allowed, the metallicity would be reduced by dilution, and an increase in $n Q$ would be required to attain a given present day metallicity. Oey (2003) takes into account interstellar mixing processes in ordinary multiphase ISM (mainly diffusion and turbulent mixing ${ }^{7}$ ), which does lead to lower metallicities within the superbubble. As a result of this analysis, turbulent mixing would appear to be more efficient than diffusion, but the lowered metallicity for parent enrichment events requires more evolution (higher $n Q$ ) to match the observed metallicities, and this in turn implies that the system's MDF drops off too steeply to match the data in the high metallicity tail of the Galactic halo MDF. Furthermore, the Population III stellar fraction is too high compared to the observations, suggesting that a discrepancy remains between the model and the observations. Future developments in the model are eagerly anticipated.

\footnotetext{
${ }^{6}$ The scatter in chromium and manganese does not increase (observationally) with decreasing iron abundance, while the observed scatter in nickel exceeds that predicted by their models.

${ }^{7}$ Note though that mixing between discrete enrichment regions is not allowed in Oey's (2003) prescription.
}

\subsection{Tsujimoto, Shigeyama, \& Yoshii (1999)}

Under the Tsujimoto et al. (1999) formalism, halo star formation is confined to separate clouds of mass $M_{c}$. Each cloud is initially composed of Population III stars, with mass fraction $x_{I I I}$, and gas that has yet to form stars. Subsequent generations of stars are assumed to form in SN remnant (SNR) shells. The mass fraction of each shell that turns into stars $(\epsilon)$ is assumed to be constant. Heavy elements ejected from a SN event are assumed to be trapped and well-mixed within the SNR shell. Some of these elements go into stars forming the next generation. This process continues until remnants are no longer capable of sweeping up sufficient gas to form shells. The mass of a shell $M_{s h}(m, t)=M_{e j}(m)+M_{s w}(m, t)$, where $M_{e j}(m)$ is the mass of the SN ejecta, and $M_{s w}(m, t)$ is the mass swept up by a shell (assumed to be $6.5 \times 10^{4} \mathrm{M}_{\odot}$ throughout their analysis).

The free parameters of the model are the mass fraction $x_{I I I}$ of metal free Population III stars initially formed in each cloud and the mass fraction $\epsilon$ of stars formed in each SNR. These values are chosen to reproduce the observed $[\mathrm{Fe} / \mathrm{H}]$ distribution function of halo field stars for $[\mathrm{Fe} / \mathrm{H}]<-1$. If $x_{I I I}$ is too large, the total gas swept up by the first SNRs exceeds the entire amount of available gas, and the star formation stops at the first or second generation. If the process is to continue as a sequence of $\mathrm{SN}$-induced star formation, $x_{I I I}$ must be $\lesssim 10^{-2}$ and $\epsilon$ confined to a narrow range such that of order one massive star is born from each SNR. If $\epsilon$ is too high, star formation soon stops with little enrichment. If $\epsilon$ is too low, star formation will proceed until most of the gas is used up, with an excess of enrichment.

Tsujimoto et al. (1999) conclude that the probability $p_{I I I}$ of observing a Population III star amongst the general background field of halo stars, under the assumption that $M_{c}=10^{6}-10^{7} \mathrm{M}_{\odot}$, should be $10^{-3}-10^{-4}$, consistent with current observational limits (Beers 2000). Their IGCE model also naturally recovers the frequency distribution of stars in the $[\mathrm{Eu} / \mathrm{Fe}]-[\mathrm{Fe} / \mathrm{H}]$ plane; $[\mathrm{Eu} / \mathrm{Fe}]$ spans $>2$ dex for $[\mathrm{Fe} / \mathrm{H}]<-2$, converging to a plateau by $[\mathrm{Fe} / \mathrm{H}] \approx-1$. Further enhancements and applications of the model are described in Suzuki \& Yoshii (2001), Tsujimoto \& Shigeyama (2002), and Tsujimoto, Shigeyama, \& Yoshii (2002).

\section{Chemodynamical Models}

GCE is intimately related to the Galactic star formation history, and star formation is equally linked to the dynamical evolution of the Galaxy. The self-consistent treatment of the chemical and dynamical evolution of a system has long been recognised as desirable, but the computationally intensive nature of the simulations made this desire difficult to realise. Advances in hardware and numerical methods over the past decade though has finally allowed chemodynamical codes to realise their theoretical promise (e.g. Larson 1976; Samland, Hensler, \& Theis 1997; Carraro, Lia, \& Chiosi 1998; Nakasato \& Nomoto 2003; 
Brook et al. 2003a). Recent three-dimensional chemodynamical evolution codes are now being applied routinely to study disk galaxies such as the Milky Way. For example, Steinmetz \& Muller (1995) succeeded in distinguishing the chemical properties between halo, bulge, and disk stars (see also Bekki \& Chiba 2001). Raiteri, Villata, \& Navarro (1996) and Berczik (1999) took into account metal enrichment by both Type II and Type Ia SNe, reproducing the correlation between $[\mathrm{O} / \mathrm{Fe}]$ and $[\mathrm{Fe} / \mathrm{H}]$ for stars in the solar neighbourhood. Brook, Kawata, \& Gibson (2003b) studied the metal enrichment from intermediate mass stars and discussed how the distribution of carbon, nitrogen, and oxygen abundances in the solar neighbourhood constrains the shape of the IMF (see also Gibson \& Mould 1997). Coupling chemodynamical models to spectrophotometric codes (see Section 6) allows one to constrain, for example, the zero point of the TullyFisher relation (e.g. Steinmetz \& Navarro 1999; Navarro \& Steinmetz 2000; Koda, Sofue, \& Wada 2000; Abadi et al. 2003a).

We now review the methodology of chemodynamical codes and their application in modelling the evolution of the Milky Way. The discussion is framed primarily within the context of our N-body/Smoothed Particle Hydrodynamics software package GCD+ (Kawata 1999; Kawata \& Gibson 2003a, 2003b; Brook et al. 2003a, 2003b).

\subsection{Brief Introduction to GCD+}

GCD+ was originally (Kawata 1999; Kawata \& Gibson 2003a) based upon Katz, Weinberg, \& Hernquist's (1996) TreeSPH code, combining the tree algorithm (Barnes \& Hut 1986) for the computation of the gravitational forces, with the smoothed particle hydrodynamics (SPH) (Lucy 1977; Gingold \& Monaghan 1977) approach to numerical hydrodynamics. The dynamics of the dark matter (DM) and stars is calculated by the N-body scheme, and the gas component is modelled using SPH. It is fully Lagrangian, three-dimensional, and highly adaptive in space and time owing to individual smoothing lengths and individual time steps. Moreover, it includes self-consistently important physical processes, such as self-gravity, hydrodynamics, radiative cooling, star formation, $\mathrm{SNe}$ feedback, and metal enrichment.

Metallicity-dependent radiative cooling is taken into account, following Sutherland \& Dopita (1993). The cooling rate for a gas with solar metallicity is larger than that for gas of primordial composition by more than an order of magnitude. As such, the cooling provided by metals should not be ignored in numerical simulations of galaxy formation (Kay et al. 2000).

GCD+ takes into account the energy feedback and metal enrichment from both $\mathrm{SNe}$ II and $\mathrm{SNe}$ Ia, as well as enrichment from ILMS. We assume that each massive $\operatorname{star}\left(\geq 8 \mathrm{M}_{\odot}\right)$ explodes as a Type II supernova. The SNe Ia rates are calculated using the model proposed by Kobayashi, Tsujimoto, \& Nomoto (2000). The yields of SNe II, SNe Ia, and ILMS are taken from Woosley \& Weaver (1995), Iwamoto et al. (1999), and van den Hoek \&
Groenewegen (1997), respectively. The mass, energy, and heavy elements are smoothed over the neighbouring gas particles using the SPH smoothing algorithm. For example, when the $i$-th star particle ejects a mass of $M_{\mathrm{SN}, i}$, a mass increment is applied to the $j$-th neighbour gas particle, as such:

$$
\Delta M_{\mathrm{SN}, j}=\frac{m_{j}}{\rho_{\mathrm{g}, i}} M_{\mathrm{SN}, i} W\left(r_{i j} / h_{i}\right)
$$

where

$$
\rho_{\mathrm{g}, i}=\left\langle\rho_{\mathrm{g}}\left(\boldsymbol{x}_{i}\right)\right\rangle=\sum_{j \neq i} m_{j} W\left(r_{i j} / h_{i}\right),
$$

and $W(x)$ is an SPH kernel (Kawata \& Gibson 2003a). GCD + monitors the evolution of all relevant chemical elements self-consistently with the dynamics of the gas, stars, and dark matter. The photometric evolution of the stellar populations is also calculated simultaneously via the Kodama \& Arimoto (1997) spectral synthesis package.

\subsection{Disk Galaxy Formation in ACDM Cosmologies}

We present now a sample disk galaxy formation simulation, undertaken within a $\Lambda$-dominated cold dark matter $(\Lambda \mathrm{CDM})$ cosmological framework, in order to demonstrate the capabilities of chemodynamical codes such as GCD + . We carried out a series of high-resolution simulations within a $\Lambda$ CDM cosmology $\left(\Omega_{0}=0.3, \Lambda_{0}=0.7\right.$, $\left.\Omega_{\mathrm{b}}=0.019 h^{-2}, h=0.7, \sigma_{8}=0.9\right)$. We used a multiresolution technique (described below) to achieve high resolution in the region of interest, including the tidal forces from large scale structures. The multi-resolution initial conditions were constructed using Bertschinger's (2001) publicly available software GRAFIC2. First, a low-resolution N-body only simulation of a comoving $20 h^{-1} \mathrm{Mpc}$ diameter sphere was performed; the mean separation of the particles therein was $20 h^{-1} / 64 \mathrm{Mpc}$. The mass of each particle was $3.63 \times 10^{9} \mathrm{M}_{\odot}$, and a fixed softening of $18.0 \mathrm{kpc}$ was applied. Next, at redshift $z=0$, we selected an $\sim 10 \mathrm{Mpc}$ diameter spherical region which contains several galaxy-sized DM halos. We traced the particles which fall into the selected region back to the initial conditions at $z=43.5$ and identified the volume which consists of those particles. Within this arbitrarily shaped volume, we replaced the low resolution particles with particles a factor of 64 times less massive. The initial density and velocities for the less massive particles are selfconsistently calculated by GRAFIC2, taking into account the density fields of the lower resolution region. Finally, we re-simulated the full volume $\left(20 h^{-1} \mathrm{Mpc}\right.$ sphere), but now including all gas dynamics, cooling, and star formation. ${ }^{8}$ The surrounding low-resolution region contributes to the high-resolution region only through gravity. The mass and softening length of individual gas (dark matter) particles in the high-resolution region were $7.33 \times 10^{6}$ $\left(4.94 \times 10^{7}\right) \mathrm{M}_{\odot}$ and $1.14(2.15) \mathrm{kpc}$, respectively.

\footnotetext{
${ }^{8}$ The gas component though was included only within the highresolution region.
} 


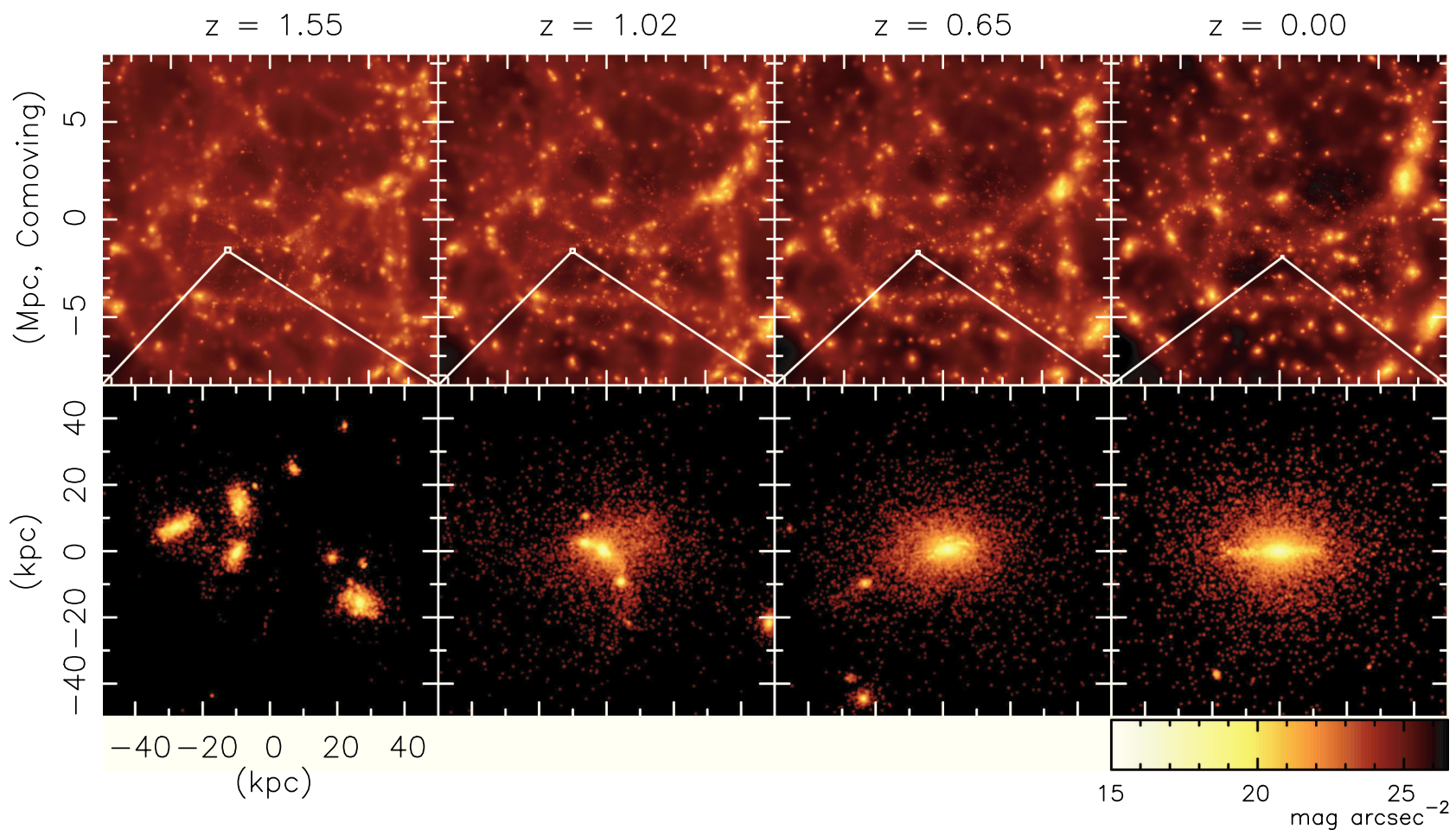

Figure 5 Dark matter density map of a portion of the $17 \mathrm{Mpc}$ (comoving) simulation volume (upper panels) and predicted $J$-band (AB magnitudes) image (physical scale) of the target galaxy (lower panels), over the redshift range $z=1.55$ to $z=0$. The projection in the lower panels has been chosen in order to view the target galaxy edge-on at $z=0$.

At $z=0$, using a friends-of-friends methodology, we identified six stellar systems which consisted of more than 2000 star particles. Two of these systems resembled large disk-like systems with kinematics consistent with rotational support; one was chosen as the target galaxy. The total virial mass of this galaxy is $\sim 2.4 \times 10^{12} \mathrm{M}_{\odot}$, and there exists a companion with $\sim 30 \%$ the mass of the target at a galactocentric distance of $\sim 270 \mathrm{kpc}$. The virial mass is defined as the mass within the virial radius, which itself is the radius of a sphere containing a mean density of $178 \Omega_{0}^{0.45}$ times the critical values $\left(\rho_{\text {crit }}=3 \mathrm{H}_{0} / 8 \pi G\right)$, after Eke, Navarro, \& Frenk (1998).

Figure 5 shows the morphological evolution of the dark matter in a central portion of the simulation volume, and the evolution of the stellar component in a $200 \mathrm{kpc}$ region centred on the target galaxy. The lower panels correspond to the predicted $J$-band (in the rest frame) image of the target galaxy. In our simulations, the star particles each carry their own age and metallicity 'tag', due to the selfconsistent chemodynamical nature of the calculation. This enables us to generate an optical to near infrared spectral energy distribution for the target galaxy (here, using the Kodama \& Arimoto 1997 spectral synthesis package).

The spectral energy distribution of each star particle is assumed to be that of a simple stellar population, i.e. a coeval and chemically homogeneous assembly of stars. We take into account the $k$-correction, but do not consider here the effects of dust absorption. Figure 5 demonstrates that the galaxy forms through conventional hierarchical clustering before $z=1$; the disk has subsequently been built up smoothly.
Figure 6 demonstrates the time evolution of the phase space information of stars within a satellite which accretes onto the central galaxy at $z \sim 0.3$. At $z=0.34$, the tidal stream of the satellite appears. The stream is identifiable in the radial velocity $\left(V_{\mathrm{R}, \mathrm{gal}}\right)$-galactocentric radius $\left(R_{\mathrm{gal}}\right)$ diagram, in addition to the $V_{\mathrm{R}, \mathrm{gal}}$-rotational velocity $\left(V_{\phi, \text { gal }}\right)$ diagram. This satellite is completely disrupted at $z=0$, and the $V_{\mathrm{R}, \mathrm{gal}}-R_{\mathrm{gal}}$ information of the member star particles of the satellite also disappears. However, in the $V_{\mathrm{R}, \mathrm{gal}}-V_{\phi \text {,gal }}$ diagram, the distribution of the member star particles is similar to that at $z=0.34$. We confirmed that the velocity phase space information of the member stars is conserved very well, even after the satellite is spatially disrupted (see also Helmi \& White 1999; Helmi \& de Zeeuw 2000).

It is worth noting however that it is difficult to identify the member stars of the accreted satellites in the velocity phase space diagram of the full sample of observed stars. The red dots in Figure 6 represent the halo stars. Here, we define the star particles with $[\mathrm{Fe} / \mathrm{H}]<-0.6$ (Chiba \& Beers 2000) as the halo stars. Figure 6 shows that in the velocity phase space diagram, the distribution of the satellite member stars overlaps that of the halo stars. Thus, to identify the member stars of the accreted satellites, additional observational information is required. Since the member stars formed within a small galaxy, it is anticipated that they might each inherit a unique chemical 'fingerprint' (Freeman \& Bland-Hawthorn 2002). Hence, the combination of such chemical tags and kinematics can be a powerful tool to identify the field stars which orginated within now accreted satellites. Comparing such 

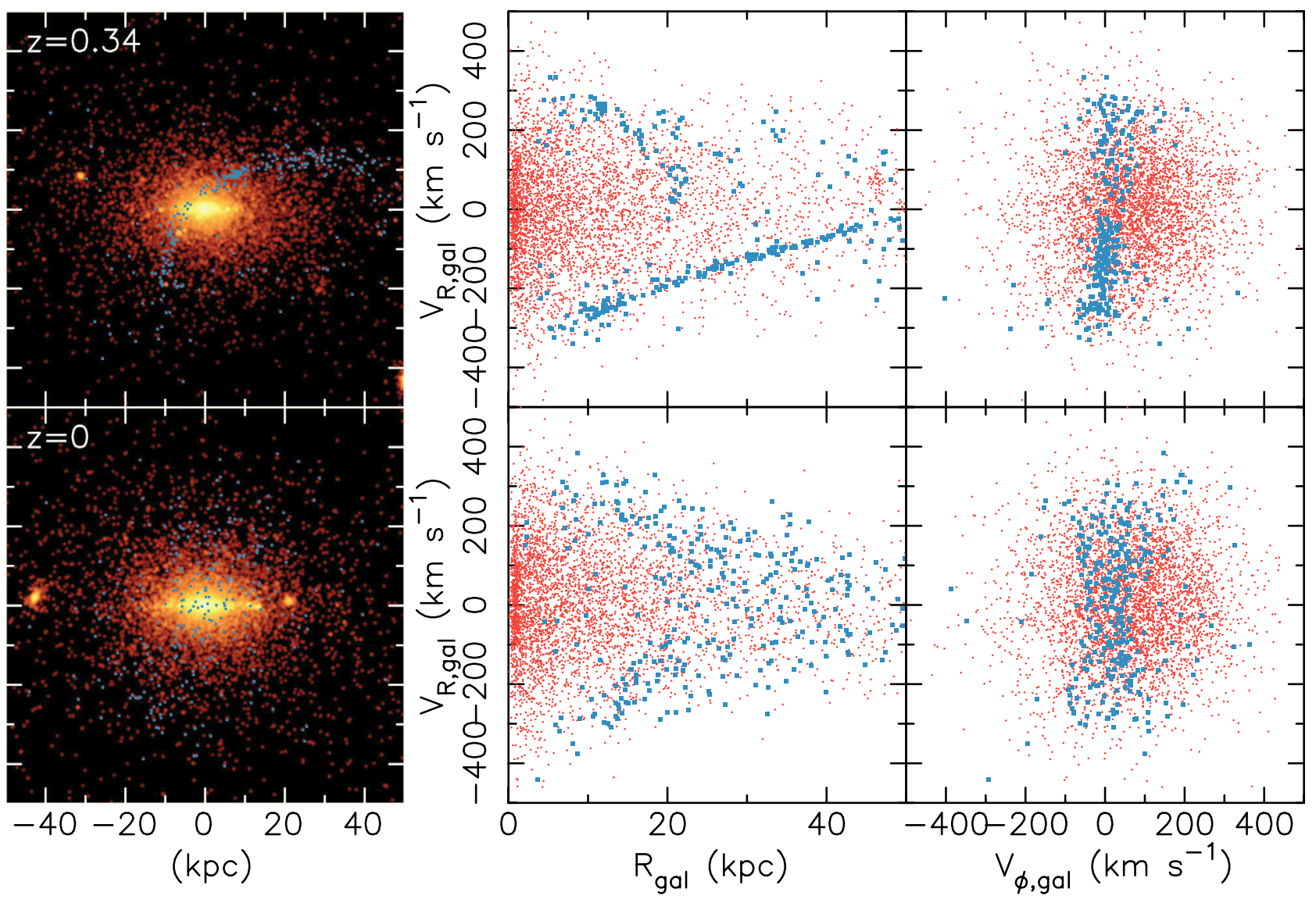

Figure 6 Snapshots of the spatial and velocity distributions of the member star particles (blue dots) of a satellite which accretes onto the central galaxy at $z \sim 0.3$. Left panels show the edge-on projection of the accreting satellite as well as $J$-band (AB magnitudes) images of the target galaxy (colour contour image, with levels as in Figure 5). Middle and right panels show the radial velocity versus the galactocentric radius (middle) and circular velocity (right) of particles with galactocentric radius less than $50 \mathrm{kpc}$. The red dots show star particles with $[\mathrm{Fe} / \mathrm{H}]<-0.6$, representative of the population of halo stars in the central galaxy.

observations and the results of chemodynamical simulations will be critical to understanding the formation history of the Milky Way. Unfortunately, current numerical simulations still struggle to overcome the classical 'overcooling problem' (White \& Frenk 1991), the signatures of which are a high-redshift star formation rate in excess of that observed, and stellar halos which are both too massive and too metal-rich (Brook et al. 2003a, 2003b; Helmi et al. 2003). The exact physical mechanism required to solve this problem remains uncertain, although a framework predicated upon an enhanced supernova feedback efficiency is one leading candidate (Navarro \& Steinmetz 2000; Brook et al. 2004).

\section{Spectrophotometry}

Determining the epoch of galaxy formation and understanding the consequent chemical evolution are amongst the fundamental quests of modern cosmology. A complementary approach to addressing the chemical evolution of galaxies is via the use of spectrophotometry. One can directly derive the age and metallicity of a galaxy, and their respective gradients therein, by comparing its observational integrated colours and/or its spectral line indices with theoretical predictions from stellar population synthesis techniques. The integrated properties of star clusters around a galaxy can also be used to induce their host galaxy's chemical evolutionary path.

Necessary ingredients for theoretical spectrophotometric predictions include stellar evolutionary tracks, isochrones, and a corresponding stellar atmosphere library which covers a wide range of stellar parameters, such as metallicity, temperature, and surface gravity. The stellar population synthesis models presented below, for example, are based upon the $Y^{2}$ isochrones ${ }^{9}$ (Kim et al. 2002) with $[\alpha / \mathrm{Fe}]=+0.3$, coupled to the post-red giant branch stellar evolutionary tracks of Yi, Demarque, \& Kim (1997). The stellar library of Lejeune, Cuisinier, \& Buser (1998) was taken for the conversion from theoretical quantities to observable quantities.

Both age-sensitive and metallicity-sensitive spectrophotometric quantities are initially constructed for a grid of simple stellar populations, for a range of age and metallicity. Composite stellar population spectrophotometric quantities can then be generated by convolving any given star formation history (with the requisite

\footnotetext{
${ }^{9}$ http://csaweb.yonsei.ac.kr/ kim/yyiso.html
} 
self-consistent treatment of chemical enrichment) with the grid of simple stellar population results (weighted by the number of stars populating each stellar evolutionary stage of each simple stellar population). The spectrophotometric quantities calculated from these composite populations can then be compared directly with observational data, and the age and metallicity of the underlying stellar population extracted. Once we have a fair selection of sample galaxies in terms of age and metallicity, we are able to investigate the detailed abundance properties along the age sequence to understand the chemical evolution of galaxies.

Figure 7 demonstrates the use of spectral index versus index plots as tools for estimating metallicity. A sample of integrated spectra for 12 Milky Way globular clusters is shown (from Cohen, Blakeslee, \& Ryzhov 1998), which was used to define a grid of Lick indices which are then compared with our recent models (H.-c. Lee \& B.K. Gibson, in preparation). The $[\mathrm{MgFe}]$ index is defined as $\sqrt{M g b \times\langle F e\rangle}$, where $\langle F e\rangle$ is $(\mathrm{Fe} 5270+\mathrm{Fe} 5335) / 2$. It is found that the metallicity $[\mathrm{Fe} / \mathrm{H}]$ that is acquired independently from the Harris (1996, February 2003 Version) compilation is surprisingly well recovered. This kind of calibration is necessary and should be quite useful for the derivation of metallicity for extragalactic star clusters and/or external galaxies that are not resolved into individual stars.

Figure 8 shows the importance of the realistic manifestation of horizontal-branch (HB) morphologies in the stellar population synthesis models for relatively old stellar systems ( $\tau>8$ Gyr) (Lee, Yoon, \& Lee 2000; Lee, Lee, \& Gibson 2002). It appears that models with a proper treatment of blue HB stars reproduce the differences between inner and outer halo clusters, ${ }^{10}$ in the sense that the inner halo clusters are not only more tightly grouped along the isochrone than the more scattered outer halo counterparts, but also relatively older. This is interesting in that the outer halo clusters with the wider range of colours at a given metallicity may indicate a different chemical 'origin', perhaps from different environments such as satellite dwarf galaxies.

Another important parameter that controls spectrophotometric quantities, as well as the mass-to-luminosity (M/L) ratio, is the IMF. A top-heavy IMF leads to highly efficient chemical enrichment due to a preponderance of Type II SNe. Conversely, chemical enrichment is minimised under the adoption of a bottom-heavy IMF. A recent attempt by Fuchs (2002) to set constraints on the mass of the disks of low surface brightness (LSB) galaxies by employing density wave theory is intriguing in this respect. Fuchs used a sample of five LSB galaxies with clear spiral structure to claim that each possesses surprisingly high stellar mass-to-luminosity ratios in the R-band $\left(M / L_{R} \geq 3\right)$, in addition to their blue colours

\footnotetext{
${ }^{10}$ The inclusion of blue HB stars also led Lee et al. $(2000,2002)$ to suggest that giant elliptical galaxies may be $\sim 1-3$ billion years older than the Milky Way.
}

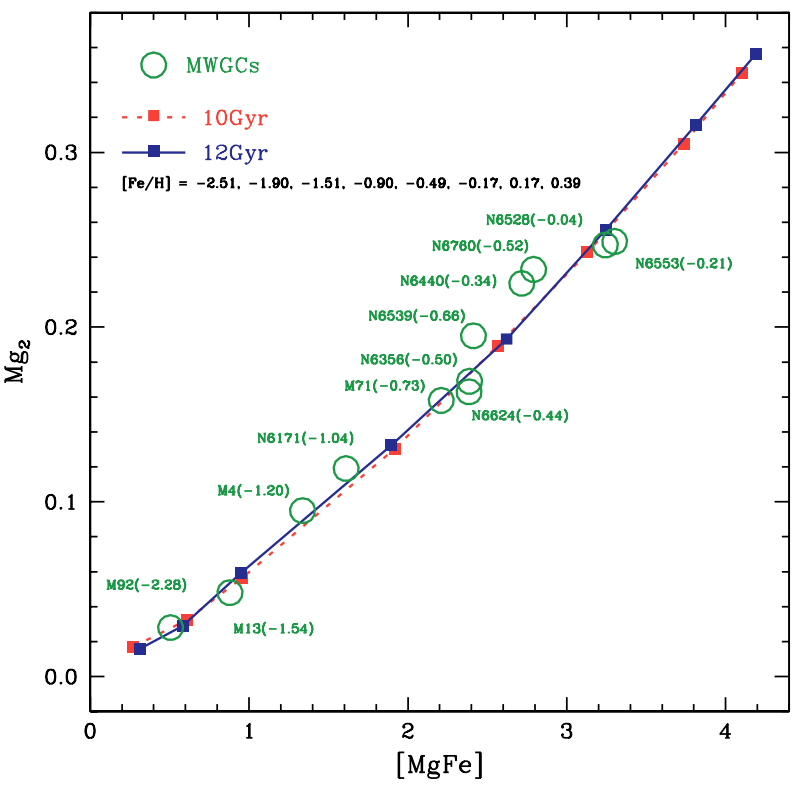

Figure 7 The metallicity [Fe/H] of Milky Way globular clusters are well-reproduced from this spectral index-index plot. The data shown - including $\mathrm{Mg}_{2}$ and $[\mathrm{MgFe}]$ indices — are taken from Cohen et al. (1998) and the metallicities in parentheses are from Harris (1996, February 2003 Version).

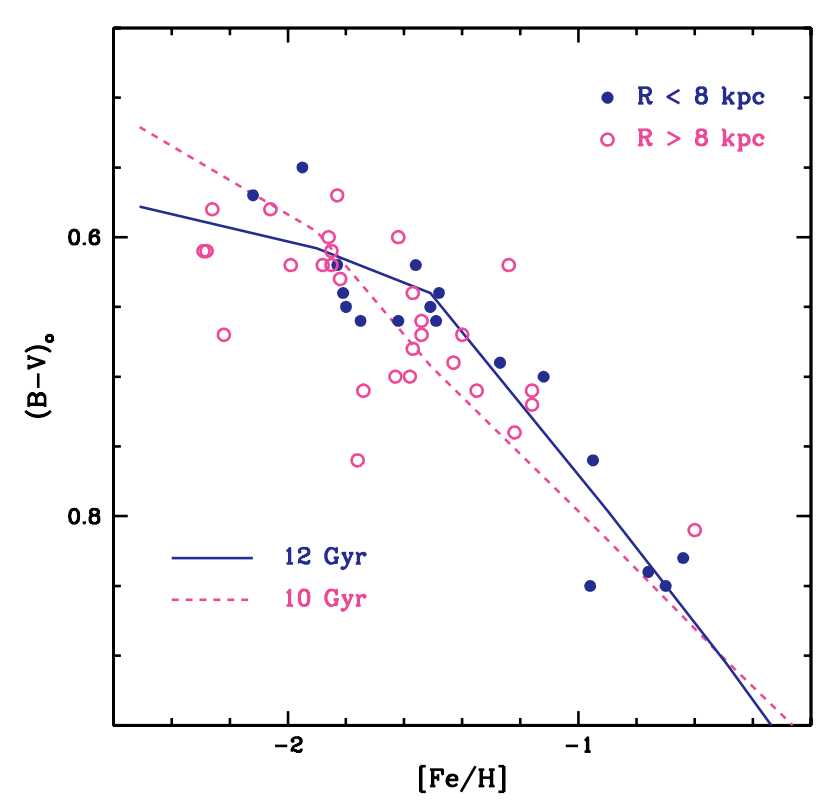

Figure 8 The relatively low-reddened Milky Way globular clusters $[E(B-V)<0.2]$ are used to calibrate our models in the $(B-V)_{o}$ versus $[\mathrm{Fe} / \mathrm{H}]$ plane. The dashed and solid lines represent ages of $10 \mathrm{Gyr}$ and $12 \mathrm{Gyr}$, respectively. Filled (open) circles correspond to inner (outer) halo globulars.

$\left((B-R)_{o}<1.0\right)$. Figure 9 demonstrates that the range of colours and mass-to-luminosity ratios spanned by the Fuchs LSB sample are consistent with a low-metallicity $([\mathrm{Fe} / \mathrm{H}] \lesssim-1.5)$, recent $(\lesssim 2 \mathrm{Gyr})$ burst of star formation, under the assumption of an IMF significantly steeper than that of Salpeter (1955). 


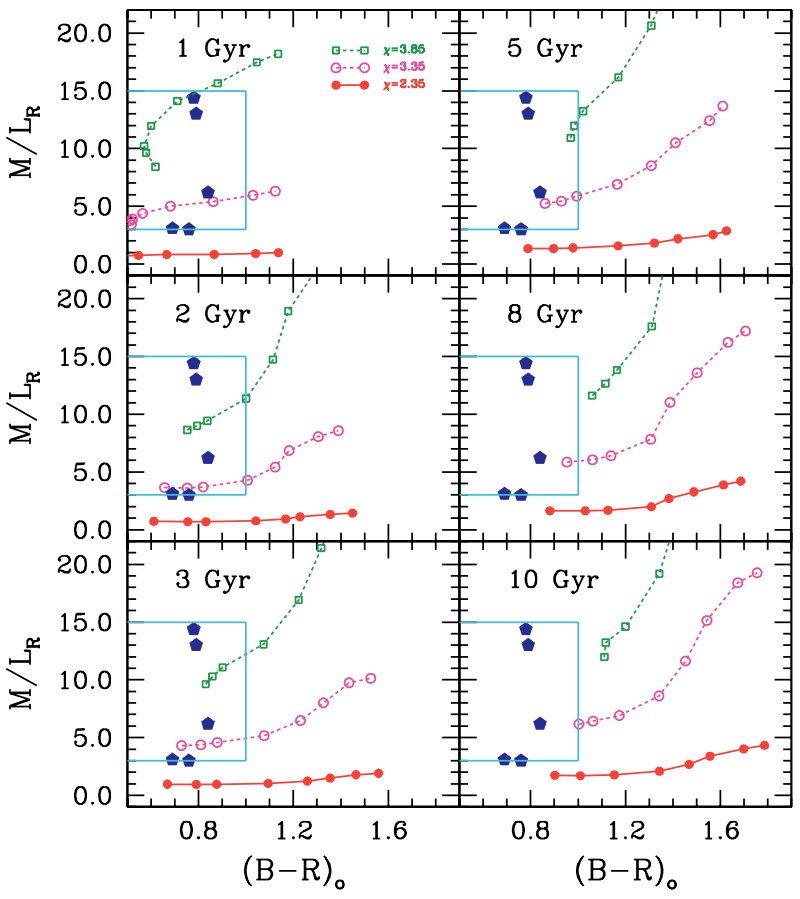

Figure 9 Our simple stellar population predictions for $(B-R)_{o}$ versus $M / L_{R}$ as a function of age for three IMF slopes. The solid line with filled circles corresponds to the standard Salpeter (1955) IMF (by number) with an exponent $\chi=2.35$. The dotted lines with open circles and open squares are $\chi=3.35$ and $\chi=3.85$ cases, respectively. At a given age and IMF exponent, the metallicity $[\mathrm{Fe} / \mathrm{H}]$ is $-2.51,-1.90,-1.51,-0.90,-0.49,-0.17,+0.17$, and +0.39 , from left to right. The filled pentagons are Fuchs (2002) sample of LSB galaxies. From the left panel, it is suggested that these LSB galaxies with high $M / L_{R}$ ratios and blue colours $\left((B-R)_{o}<1.0\right)$ may be consistent with a relatively low metallicity and rather recent $(\lesssim 2 \mathrm{Gyr}$ ago) star formation, by introducing a steep IMF (Lee et al. 2004).

\section{Future Directions}

Any model for the formation and evolution of the Milky Way is only as good as the observational data upon which it is calibrated. We wish to end this review with an outline of some of the exciting observational programs which will come to fruition over the coming decade. Each of these datasets is capable of constraining - in a new and significant manner - the GCE models discussed in Sections 3-6.

We are fortunate that for the Milky Way we can, in principle, obtain full six-dimensional phase space (spatial and kinematical) and chemical information, for individual stars. Such data provides unique insights into the detailed formation history of our Galaxy (e.g. Eggen, LyndenBell, \& Sandage 1962; Chiba \& Beers 2000; Bekki \& Chiba 2001; Freeman \& Bland-Hawthorn 2002; Brook et al. 2003a). The discovery of the disrupting Sagittarius dwarf (Ibata, Gilmore, \& Irwin 1994) and other halo substructure signatures (Helmi et al. 1999; Chiba \& Beers 2000; Gilmore, Wyse, \& Norris 2002; Brook et al. 2003a) has also demonstrated the value of stellar kinematics in reconstructing satellite accretion events. Such events can (potentially) aid in determining the fraction of the halo which was accreted and the fraction which formed in situ (Helmi \& White 1999; Helmi \& de Zeeuw 2000; Harding et al. 2001).

We identify three landmark projects which will advance significantly the field of 'Galactic Archaeology':

- RAVE ${ }^{11}$ (RAdial Velocity Experiment) is an ambitious all-sky survey (complete to $\mathrm{V}=16$ ) aimed at measuring the radial velocities (with precision $\lesssim 2 \mathrm{~km} \mathrm{~s}^{-1}$ ), metallicities, and abundance ratios (both to $\sim 0.1$ dex precision) of 50 million stars using the United Kingdom Schmidt Telescope (UKST), together with a northern counterpart, over the period 2006-2010, providing a vast stellar kinematic and chemical database. A twoyear pilot survey commenced on the UKST in April 2003, making use of the existing 6dF Facility. This pilot survey will obtain comparable quality data to the main survey for $10^{5}$ stars (spanning $\sim 8500 \mathrm{deg}^{2}, 9<\mathrm{I}<12$ ), of which approximately half have accurate Tycho- 2 proper motions.

- ESA's GAIA ${ }^{12}$ satellite mission, scheduled for launch in 2010, will conduct a census of $\sim 10^{9}$ stars $-\sim 100$ revisits per star over a five-year period - measuring positions of all objects down to $\mathrm{V}=20$. Positional accuracies of $\sim 4 \mu$ as at $\mathrm{V}=10$ and $\sim 10 \mu$ as at $\mathrm{V}=15$ will yield distances accurate to $10 \%$ at heliocentric distances of $\sim 10 \mathrm{kpc}$. Radial velocity accuracy comparable to that of RAVE is expected (also to $\mathrm{V}=16$ ).

- NASA's Space Interferometry Mission - SIM, ${ }^{13}$ scheduled for launch in 2009, complements GAIA's $4 \mu$ as positional accuracy with a narrow-angle mode allowing accuracies of $\sim 1 \mu$ as.

There currently exists little compelling evidence that a massive $\left(>10{ }^{10} \mathrm{M}_{\odot}\right)$ satellite has been accreted by the Milky Way within the past $\sim 10 \mathrm{Gyr}$ (Gilmore et al. 2002). ${ }^{14}$ Instead, later accreted satellites are more likely to resemble dwarf systems such as the Local Group's dwarf spheroidals (dSphs). Recent (and spectacular) highresolution spectroscopic observations of individual stars in dSphs have demonstrated that their ratio of $\alpha$-elements to iron tend to be close to solar (e.g. Shetrone, Côté, \& Sargent 2001; Shetrone et al. 2003). This elemental abundance pattern is not reflected in the present-day halo field stars - a somewhat surprising result considering the aforementioned kinematical evidence for halo accretion events. An inescapable conclusion to be drawn from these data is that the present-day Local Group dSphs are not the primary 'stellar donors' to the Galactic halo (Tolstoy et al. 2003). Whether such accreted satellites are responsible for the thick disk though remains an intriguing possibility (Bekki \& Chiba 2002; Abadi et al. 2003b). Chemical 'tagging' of Local Group dSphs is still very much in its infancy,

\footnotetext{
${ }^{11} \mathrm{http} / / /$ astronomy.swin.edu.au/RAVE/

$12 \mathrm{http}$ ///astro.estec.esa.nl/GAIA/

13 http://sim.jpl.nasa.gov/

14 The potential impact that such a massive satellite would have upon the disk supports this contention (Kawata, Thom, \& Gibson 2003).
} 
but a burgeoning field which will see enormous increases in sample sizes over the coming few years, thanks to substantial investments of $8 \mathrm{~m}$-class time.

The hunt for the most primitive stars in the Milky Way has led to some of the most exciting discoveries in recent astronomy, including the detection of a star with an iron abundance less than 1/200000 that of the Sun (Christlieb et al. 2002). Having formed from almost pristine primordial gas, this star (HE0107-5240) is a nearby counterpart to the high-redshift universe and provides insight into the earliest epochs of Galaxy formation. Two factors could prohibit the detection of a bona fide zerometallicity Population III star: (1) metal-free gas might favour the formation of higher mass stars with short lifetimes, such that there are no surviving Population III stars today (Nakamura \& Umemura 2001); and (2) the surfaces of Population III stars may have been polluted with metals either from internal processing or through the accretion of interstellar matter (Shigeyama et al. 2003). Despite their paucity, thousands of candidate metal-poor stars have been selected for follow-up spectroscopy by programs such as the HK and Hamburg/ESO surveys (Beers 2000). The tally of $\sim 100$ stars with $[\mathrm{Fe} / \mathrm{H}]<-3$ found by the HK survey is expected to grow by more than a factor of five with the Hamburg/ESO program (Christlieb 2003). As alluded to in Section 4, the abundance pattern in extremely metalpoor stars may reflect the chemical fingerprint of a single Population III star and provides empirical constraints on models of 'The First Stars' (e.g. Umeda \& Nomoto 2003). A project using the Sloan Digital Sky Survey dataset to find metal-poor stars in the halo and thick disk of the Milky Way is also underway (Allende Prieto et al. 2003).

We have only touched the 'tip of the iceberg' here - the combination of 'big glass' and 'big surveys' over the next decade will see an explosion of chemo-kinematical data coming on-line. ${ }^{15}$ Such a wealth of impending information makes this the optimal time to be a theorist interested in understanding the detailed formation and evolution of our Milky Way Galaxy.

\section{Acknowledgements}

We thank Chris Flynn, Stefan Harfst, Chris Brook, Chris Thom, Tim Connors, Alexander Knebe, Mike Beasley, Antonio Pipino, Alessandro Chieffi, Marco Limongi, John Lattanzio, and Amanda Karakas, for helpful discussions. We acknowledge the Yukawa Institute Computer Facility, the Astronomical Data Analysis Centre of the National Astronomical Observatory Japan, and the Victorian and Australian Partnerships for Advanced Computing, for the use of their facilities during the preparation of this review. This work has benefited from the ongoing financial support of the Australian Research Council, to whom we are indebted.

\footnotetext{
${ }^{15}$ Indeed, the data 'glut' that surveys like RAVE will contribute to is one motivation driving the development of virtual observatories.
}

\section{References}

Abadi, M. G., Navarro, J. F., Steinmetz, M., \& Eke, V. R. 2003a, ApJ, 591, 499

Abadi, M. G., Navarro, J. F., Steinmetz, M., \& Eke, V. R. 2003b, ApJ, 597, 21

Alibés, A., Labay, J., \& Canal, R. 2001, A\&A, 370, 1103

Allende Prieto, C., Lambert, D. L., \& Asplund, M. 2001, ApJ, 556, L63

Allende Prieto, C., Beers, T. C., Li, Y., Newberg, H. J., Wilhelm, R., \& Yanny, B. 2003, Carnegie Observatories Astrophysics Series, Volume 4: Origin and Evolution of the Elements, eds. A. McWilliam \& M. Rauch (Pasadena: Carnegie Observatories), http://www.ociw.edu/ociw/symposia/series/symposium4/ proceedings.html

Anders, E., \& Grevesse, N. 1989, GeCoA, 53, 197

Argast, D., Samland, M., Gerhard, O. E., \& Thielemann, F.-K. 2000, A\&A, 356, 873

Argast, D., Samland, M., Thielemann, F.-K., \& Gerhard, O. E. 2002, A\&A, 388, 842

Barnes, J. E., \& Hut, P. 1986, Nature, 324, 446

Beers, T. C. 2000, in The First Stars, eds. A. Weiss, T. G. Abel, \& V. Hill (Berlin: Springer), 3

Bekki, K., \& Chiba, M. 2001, ApJ, 558, 666

Bekki, K., \& Chiba, M. 2002, ApJ, 566, 245

Berczik, P. 1999, A\&A, 348, 371

Bertschinger, E. 2001, ApJS, 137, 1

Boissier, S., \& Prantzos, N. 2000, MNRAS, 312, 398

Brook, C. B., Kawata, D., Gibson, B. K., \& Flynn, C. 2003a, ApJL, 585,125

Brook, C. B., Kawata, D., \& Gibson, B. K. 2003b, MNRAS, 343, 913

Brook, C. B., Kawata, D., Gibson, B. K., \& Flynn, C. 2004, MNRAS, in press

Buser, R., Rong, J., \& Karaali, S. 1998, A\&A, 331, 934

Carraro, G., Lia, C., \& Chiosi, C. 1998, MNRAS, 297, 1021

Chen, B., et al. 2001, ApJ, 553, 184

Chiappini, C., Matteucci, F., \& Gratton, R. 1997, ApJ, 477, 765

Chiappini, C., Matteucci, F., \& Padoan, P. 2000, ApJ, 528, 711

Chiba, M., \& Beers, T. C. 2000, AJ, 119, 2843

Chieffi, A., \& Limongi, M. 2002, ApJ, 577, 281

Christlieb, N. 2003, RvMA, 16, 191

Christlieb, N., et al. 2002, Nature, 419, 904

Cohen, J. G., Blakeslee, J. P., \& Ryzhov, A. 1998, ApJ, 496, 808

Copi, C. J. 1997, ApJ, 487, 704

Dekel, A., \& Silk, J. 1986, ApJ, 303, 39

Dopita, M. A., \& Ryder, S. D. 1994, ApJ, 430, 163

Edvardsson, B., Andersen, J., Gustafsson, B., Lambert, D. L., Nissen, P. E., \& Tomkin, J. 1993, A\&A, 275, 101

Eggen, O. J., Lynden-Bell, D., \& Sandage, A. R. 1962, ApJ, 136, 748

Eke, V. R., Navarro, J. F., \& Frenk, C. S. 1998, ApJ, 503, 569

Feltzing, S., Holmberg, J., \& Hurley, J. R. 2001, A\&A, 377, 911

Fenner, Y., \& Gibson, B. K. 2003, PASA, 20, 189

Fenner, Y., Gibson, B. K., Lee, H.-c., Karakas, A. I., Lattanzio, J. C., Chieffi, A., Limongi, M., \& Yong, D. 2003, PASA, 20, 340

Freeman, K. C., \& Bland-Hawthorn, J. 2002, ARAA, 40, 487

Fuchs, B., 2002, in Dark Matter in Astro- and Particle Physics, eds. H. V. Klapdor-Kleingrothaus \& R. D. Viollier (Berlin: Springer), 28

Gay, P. L., \& Lambert, D. L. 2000, ApJ, 533, 260

Gibson, B. K. 1997, MNRAS, 290, 471

Gibson, B. K., \& Mould, J. R. 1997, ApJ, 482, 98

Gibson, B. K., Loewenstein, M., \& Mushotzky, R. F. 1997, MNRAS, 290,623

Gilmore, G., \& Reid, N. 1983, MNRAS, 202, 1025

Gilmore, G., Wyse, R. F. G., \& Kuijken, K. 1989, ARA\&A, 27, 555

Gilmore, G., Wyse, R. F. G., \& Norris, J. E. 2002, ApJ, 574, L39

Gingold, R. A., \& Monaghan, J. J. 1977, MNRAS, 181, 375 
Greggio, L., \& Renzini, A. 1983, A\&A, 118, 217

Harding, P., Morrison, H. L., Olszewski, E. W., Arabadjis, J., Mateo, M., Dohm-Palmer, R. C., Freeman, K. C., \& Norris, J. E. 2001, AJ, 122, 1397

Harris, W. E. 1996, AJ, 112, 1487

Helmi, A., \& de Zeeuw, P. T. 2000, MNRAS, 319, 657

Helmi, A., Navarro, J. F., Meza, A., Steinmetz, M., \& Eke, V. R. 2003, ApJ, 592, L25

Helmi, A., \& White, S. D. M. 1999, MNRAS, 307, 495

Helmi, A., White, S. D. M., de Zeeuw, P. T., \& Zhao, H. 1999, Nature, 402, 53

Holweger, H. 2001, in Solar and Galactic Composition, AIP Conference Proceedings 598, ed. R.F. Wimmer-Schweingruber (Melville, NY: AIP), 23

Ibata, R., Gilmore, G., \& Irwin, M. J. 1994, Nature, 370, 194

Ibukiyama, A., \& Arimoto, N. 2002, A\&A, 394, 927

Ikuta, C., \& Arimoto, N. 1999, PASJ, 51, 459

Iwamoto, K., Brachwitz, F., Nomoto, K., Kishimoto, N., Umeda, H., Hix, W. R., \& Thielemann, F.-K. 1999, ApJS, 125, 439

Karakas, A. I., \& Lattanzio, J. C. 2003, PASA, 20, 279

Kawata, D. 1999, PASJ, 51, 931

Kawata, D., \& Gibson, B. K. 2003a, MNRAS, 340, 908

Kawata, D., \& Gibson, B. K. 2003b, MNRAS, 346, 135

Kawata, D., Thom, C., \& Gibson, B. K. 2003, PASA, 20, 263

Katz, N., Weinberg, D. H., \& Hernquist, L. 1996, ApJS, 105, 19

Kay, S. T., Pearce, F. R., Jenkins, A., Frenk, C. S., White, S. D. M., Thomas, P. A., \& Couchman, H. M. P. 2000, MNRAS, 316, 374

Kim, Y.-C., Demarque, P., Yi, S. K., \& Alexander, D. R. 2002, ApJS, 143,499

Kobayashi, C., Tsujimoto, T., \& Nomoto, K. 2000, ApJ, 539, 26

Koda, J., Sofue, Y., \& Wada, K. 2000, ApJ, 531, L17

Kodama, T., \& Arimoto, N. 1997, A\&A, 320, 41

Kotoneva, E., Flynn, C., Chiappini, C., \& Matteucci, F. 2002, MNRAS, 336, 879

Kroupa, P., Tout, C. A., \& Gilmore, G. 1993, MNRAS, 262, 545

Larson, R. B. 1974, MNRAS, 169, 229

Larson, R. B. 1976, MNRAS, 176, 31

Lee, H.-c., Gibson, B. K., Flynn, C., Kawata, D., \& Beasley, M. A. 2004, MNRAS, submitted

Lee, H.-c., Lee, Y.-W., \& Gibson, B. K. 2002, AJ, 124, 2664

Lee, H.-c., Yoon, S.-J., \& Lee, Y.-W. 2000, AJ, 120, 998

Lejeune, T., Cuisinier, F., \& Buser, R. 1998, A\&AS, 130, 65

Lucy, L. B. 1977, AJ, 82, 1013

Maeder, A. 1992, A\&A, 264, 105

Malinie, G., Hartmann, D. H., \& Mathews, G. J. 1991, ApJ, 376,520

Malinie, G., Hartmann, D. H., Clayton, D. D., \& Mathews, G. J. 1993, ApJ, 413, 633

Matteucci, F. 2001, The Chemical Evolution of the Galaxy (Dordrecht: Kluwer)

Matteucci, F., \& Greggio, L. 1986, A\&A, 154, 279

Matteucci, F., \& Tornambé, A. 1987, A\&A, 185, 51

Matteucci, F., Raiteri, C. M., Busson, M., Gallino, R., \& Gratton, R. 1993, A\&A, 272, 421

Mishenina, T. V., Kovtyukh, V. V., Soubiran, C., Travaglio, C., \& Busso, M. 2002, A\&A, 396, 189

Nakamura, F., \& Umemura, M. 2001, ApJ, 548, 19

Nakasato, N., \& Nomoto, K. 2003, ApJ, 588, 842

Navarro, J. F., \& Steinmetz, M. 2000, ApJ, 538, 477

Oey, M. S. 2000, ApJ, 542, L25

Oey, M. S. 2003, MNRAS, 339, 849

Pagel, B. E. J., \& Patchett, B. E. 1975, MNRAS, 172, 13

Pilyugin, L. S., \& Edmunds, M. G. 1996, A\&A, 313, 792
Portinari, L., \& Chiosi, C. 1999, A\&A, 350, 827

Portinari, L., \& Chiosi, C. 2000, A\&A, 355, 929

Raiteri, C. M., Villata, M., \& Navarro, J. F. 1996, A\&A, 315, 105

Recchi, S., Matteucci, F., \& D'Ercole, A. 2001, MNRAS, 322, 800

Robin, A. C., Reylé, C., Derrière, S., \& Picaud, S. 2003, A\&A, 409, 523

Rocha-Pinto, H. J., Scalo, J., Maciel, W. J., \& Flynn, C. 2000a, ApJL, 531, L1 15

Rocha-Pinto, H. J., Maciel, W. J., Scalo, J., \& Flynn, C. 2000b, A\&A, 358,850

Salpeter, E. E. 1955, ApJ, 121, 161

Samland, M., Hensler, G., \& Theis, Ch. 1997, ApJ, 476, 544

Scalo, J. M. 1986, FCPh, 11, 1

Schmidt, M. 1959, ApJ, 129, 243

Searle, L., \& Zinn, R. 1978, ApJ, 225, 357

Shetrone, M. D., Côté, P., \& Sargent, W. L. W. 2001, ApJ, 548, 592

Shetrone, M. D., Venn, K. A., Tolstoy, E., Primas, F., Hill, V., \& Kaufer, A. 2003, AJ, 125, 684

Shigeyama, T., Tsujimoto, T., \& Yoshii, Y. 2003, ApJ, 586, L57

Smartt, S. J., \& Rolleston, W. R. J. 1997, 481, L47

Smecker-Hane, T. A., \& Wyse, R. F. G. 1992, AJ, 103, 1621

Spite, M., et al. 2003, in Carnegie Observatories Astrophysics Series, Volume 4: Origin and Evolution of the Elements, eds. A. McWilliam \& M. Rauch (Pasadena: Carnegie Observatories), http://www.ociw.edu/ociw/symposia/series/symposium4/ proceedings.html

Steinmetz, M., \& Muller, E. 1995, MNRAS, 276, 549

Steinmetz, M., \& Navarro, J. F. 1999, ApJ, 513, 555

Sutherland, R. S., \& Dopita, M. A. 1993, ApJS, 88, 253

Suzuki, T. K., \& Yoshii, Y. 2001, ApJ, 549, 303

Timmes, F. X., Woosley, S. E., \& Weaver, T. A. 1995, ApJS, 98, 617

Tinsley, B. M. 1980, FCPh, 5, 287

Tolstoy, E., Venn, K. A., Shetrone, M. D., Primas, F., Hill, V., Kaufer, A., \& Thomas, S. 2003, AJ, 125, 707

Travaglio, C., Galli, D., \& Burkert, A. 2001, ApJ, 547, 217

Travaglio, C., Kifonidis, K., \& Müller, E. 2003, in Carnegie Observatories Astrophysics Series, Volume 4: Origin and Evolution of the Elements, eds. A. McWilliam \& M. Rauch (Pasadena: Carnegie Observatories), http://www.ociw.edu/ociw/ symposia/series/symposium4/proceedings.html

Tsujimoto, T., \& Shigeyama, T. 2002, ApJ, 571, L93

Tsujimoto, T., Shigeyama, T., \& Yoshii, Y. 1999, ApJ, 519, L63

Tsujimoto, T., Shigeyama, T., \& Yoshii, Y. 2002, ApJ, 565, 1011

Twarog, B. A. 1980, ApJ, 242, 242

Umeda, H., \& Nomoto, K. 2002, ApJ, 565, 385

Umeda, H., \& Nomoto, K. 2003, Nature, 422, 871

van den Hoek, L. B., \& de Jong, T. 1997, A\&A, 318, 231

van den Hoek, L. B., \& Groenewegen, M.A.T. 1997, A\&AS, 123 305

van der Kruit, P. C., \& Searle, L. 1982, A\&A, 110, 79

White, S. D. M., \& Frenk, C. S. 1991, ApJ, 379, 52

Woosley, S. E., \& Weaver, T. A. 1995, ApJS, 101, 181

Worthey, G., \& Espana, A. L. 2003, in Carnegie Observatories Astrophysics Series, Volume 4: Origin and Evolution of the Elements, eds. A. McWilliam \& M. Rauch (Pasadena: Carnegie Observatories), http://www.ociw.edu/ociw/symposia/ series/symposium4/proceedings.html

Wyse, R. F. G., \& Gilmore, G. 1988, AJ, 95, 1404

Wyse, R. F. G., \& Gilmore, G. 1993, in The Globular Cluster-Galaxy Connection, ASP Conf. Series 48, eds. G.H. Smith \& J.P. Brodie (San Francisco: ASP), 727

Yi, S., Demarque P., \& Kim, Y.-C. 1997, ApJ, 482, 677

Yoshii, Y., \& Rodgers, A. W. 1989, AJ, 98, 853 